\title{
Autoeficácia, intensidade de dor e qualidade de vida em indivíduos com dor crônica
}

\author{
Mariana Souza e Silva ${ }^{1}$, Priscilla Hortense ${ }^{2}$, Anamaria Alves Napoleão ${ }^{3}$, Thais Stefane ${ }^{4}$
}

\footnotetext{
${ }^{1}$ Enfermeira, Mestre em Enfermagem. Enfermeira da Prefeitura Municipal de São Carlos. São Carlos, SP, Brasil. E-mail: marisosil@bol.com.br.

${ }^{2}$ Enfermeira, Doutora em Enfermagem. Professora Adjunta da Universidade Federal de São Carlos (UFSCAR). São Carlos, SP, Brasil. E-mail: priscillaufscar@gmail.com.

${ }^{3}$ Enfermeira, Doutora em Enfermagem. Professora Adjunta da UFSCAR. São Carlos, SP, Brasil. E-mail: anamaria@ufscar.br.

${ }^{4}$ Enfermeira, Mestre em Enfermagem. São Carlos, SP, Brasil. E-mail:

t.stefane@gmail.com.
}

Recebido: 05/04/2014.

Aceito: 16/04/2015.

Publicado: 31/03/2016.

\section{Como citar esse artigo:}

Silva MS, Hortense $P$, Napoleão AA,

Stefane T. Autoeficácia, intensidade de dor e qualidade de vida em indivíduos com dor crônica. Rev. Eletr. Enf. [Internet]. 2016 [acesso em:_____ ];18:e1145. Disponível em:

http://dx.doi.org/10.5216/ree.v18.29308.

\section{RESUMO}

O estudo teve como objetivo correlacionar autoeficácia, qualidade de vida e intensidade de dor em pessoas com dor crônica. Estudo tipo corte transversal. Amostra de 95 pessoas com dor crônica, recrutada na área de abrangência de uma Unidade de Saúde da Família. Os instrumentos utilizados na pesquisa foram: a Escala de Autoeficácia para Dor, Escala Numérica de Dor e o WHOQOL - Bref, para avaliação da autoeficácia, intensidade de dor e qualidade de vida. As correlações indicaram que indivíduos com dor crônica e elevados níveis de autoeficácia podem apresentar menor intensidade de dor e melhor qualidade de vida. Autoeficácia e qualidade de vida são variáveis a serem consideradas na avaliação de pessoas com dor crônica.

Descritores: Dor; Medição da Dor; Autoeficácia; Qualidade de Vida; Cuidados de Enfermagem.

\section{INTRODUÇÃO}

A dor crônica persistente ocasiona desafios diários na vida das pessoas, tais como mudanças de papéis na família e na sociedade, responsabilidades e enfrentamento das consequências emocionais negativas, o que resulta em diminuição da qualidade de vida. No entanto, existem evidências de que os fatores cognitivos comportamentais como crenças de autoeficácia, catastrofização, medo, evitação e aceitação são variáveis que influenciam no enfrentamento da dor ${ }^{(1)}$. Isso poderia explicar as diferenças individuais observadas no enfrentamento da dor, juntamente com fatores genéticos, fisiológicos, culturais, espirituais e emocionais.

Crenças de autoeficácia são capazes de interferir na experiência da dor, e desta forma, se destacam como crenças a serem consideradas na avaliação de pessoas com dor crônica, uma vez que este construto tem sido apontado como fundamental no seu manejo ${ }^{(2-3)}$. 
As crenças de autoeficácia se referem às habilidades pessoais de desempenhar com sucesso determinadas tarefas ou apresentar comportamentos para produzir um resultado desejável. 0 termo "crenças" se define como ideias que consideramos como verdade; são nossas noções sobre todos os aspectos da vida, seja sobre nós mesmos, seja sobre percepções, pensamentos e até fatores ambientais ${ }^{(4)}$.

Neste sentido, as crenças, as atitudes, os valores e os comportamentos relacionados à dor são culturalmente adquiridos e podem ser modificados, por isso se torna possível o uso de conceitos psicossocioculturais no manejo de doentes com dor crônica ${ }^{(1-4)}$.

As pessoas com grau elevado de autoeficácia acreditam ser capazes de lidar com os diversos acontecimentos da vida, de vencer obstáculos, de procurar desafios e manter um alto grau de confiança na sua capacidade de obter êxito e de controlar a própria vida. Em situações em que a autoeficácia é diminuída, o inverso pode ser percebido, possibilitando baixa capacidade de resoluções dos problemas ${ }^{(4)}$. A análise das crenças de autoeficácia permite ao profissional avaliar a situação e os recursos que estão disponíveis para decidir que ações podem ser escolhidas.

Há evidências de que intervenções para a melhora da autoeficácia ocasionam melhora na qualidade de vida em pessoas com dor crônica; esta melhora tem sido relatada nos domínios físico e psicológico da qualidade de vida ${ }^{(5-6)}$

A qualidade de vida é uma variável a ser considerada na avaliação da pessoa com dor e da proposta terapêutica empregada. Qualidade de vida é definida pela Organização Mundial da Saúde como sendo a percepção do indivíduo de sua posição na vida, no seu contexto cultural e dos sistemas de valores nos quais ele vive em relação aos seus objetivos, expectativas, padrões e preocupações ${ }^{(7)}$.

Em uma pesquisa que estudou brasileiros e australianos observou-se que as crenças elevadas de autoeficácia eram um fator significativo para a diminuição de incapacidades em pessoas com dor crônica ${ }^{(8)}$. Em outro estudo realizado nas regiões sul e sudeste do Brasil observou-se que baixos níveis de crenças de autoeficácia estão relacionados com maior incapacidade e maior intensidade da dor, o que demonstra que as crenças negativas de autoeficácia parecem ser um importante preditor de incapacidade. Baseado nesta perspectiva, pode-se esperar que indivíduos com dor crônica que apresentem uma baixa autoeficácia tenham mais chances de terem estratégias de enfrentamento menos efetivas, o que poderia ocasionar incapacidade física e esforços para manterem-se empregado ou trabalhando apesar da dor ${ }^{(2)}$.

Uma revisão de literatura ${ }^{(5)}$ apontou que o ensino de habilidades para a gestão do dia a dia na convivência com a dor é uma intervenção eficaz para o seu manejo. Tais intervenções devem ser iniciadas nas pessoas que aceitam a natureza crônica da dor e que estão dispostas a autogerir o manejo da sua própria dor. Para o ensino da autogestão da dor, podem ser usadas estratégias de dinâmica de grupo, conversas individuais, intervenções online por meio do uso da rede mundial de computadores, dentre outras.

A autoeficácia emerge como uma importante variável para explicar a experiência de dor em pessoas com fibromialgia, constituindo uma variável a ser avaliada no cuidado a pessoa com dor ${ }^{(9)}$.

Autoeficácia e qualidade de vida são variáveis subjetivas a serem consideradas na avaliação das 
pessoas com dor crônica. Dessa forma, conhecer e associar variáveis como dor, autoeficácia e qualidade de vida se faz importante para a pesquisa e para o atendimento em diferentes cenários do cuidado. Assim, esse estudo teve como objetivo realizar correlacionar autoeficácia, qualidade de vida e intensidade de dor em pessoas com dor crônica.

\section{MÉTODO}

Estudo tipo corte transversal, realizado em uma área de abrangência de uma Unidade de Saúde da Família, em uma cidade do interior do sudeste brasileiro.

A Unidade de Saúde da Família contava, na época em que o estudo foi realizado, com 2.760 pessoas cadastradas. A amostra de conveniência foi constituída por meio da análise dos prontuários de todos os pacientes cadastrados. Os critérios de inclusão utilizados foram: constar, no prontuário, diagnóstico médico de doença crônica que resultasse em dor crônica, ser maior de 18 anos, apresentar relato de dor crônica com duração há mais de três meses ${ }^{(10)}$ e verbalizar disposição física e emocional para responder às questões solicitadas. $\mathrm{O}$ critério de exclusão foi o insucesso na tentativa de contato com o participante na sua residência em pelo menos duas vezes.

O presente estudo foi submetido e aprovado pelo Comitê de Ética em Pesquisa da Universidade Federal de São Carlos (UFSCar) (Parecer no 495/2010). Todos os participantes assinaram o Termo de Consentimento Livre e Esclarecido aprovado por este Comitê.

Os indivíduos foram convidados a participar da pesquisa durante a visita domiciliar programada ou no momento em que compareceu à Unidade de Saúde da Família. A coleta de dados foi realizada pelo próprio pesquisador no domicílio dos participantes ou nas dependências da unidade de saúde, conforme a preferência do participante. O período de coleta de dados foi entre julho e setembro de 2010.

Para que os objetivos fossem atingidos, os instrumentos utilizados foram: um instrumento para caracterizar os participantes no que se refere aos dados sociodemográficos (gênero, idade, cor da pele, escolaridade, renda familiar e Índice de Massa Corporal, seguindo a classificação da Organização Mundial da Saúde ${ }^{(11)}$ ) e aos aspectos relacionados à dor (localização da dor, diagnóstico médico da causa da condição dolorosa e tempo de diagnóstico; estas duas últimas informações foram retiradas do prontuário do paciente), a Escala de Autoeficácia para Dor, escala numérica de avaliação da dor e o WHOQOL - Bref.

A intensidade dolorosa foi avaliada por meio de uma escala numérica de dor de 11 pontos ( 0 a 10), sendo que o sujeito apontou seu nível de dor considerando que zero (0) significa ausência de dor, 10 significa dor insuportável e os níveis intermediários significam níveis intermediários de dor ${ }^{(12)}$.

Para avaliar as crenças de autoeficácia para dor crônica, utilizou-se a Escala de Autoeficácia para Dor Crônica, que foi desenvolvida em $1995^{(13)}$ e validada para língua portuguesa em $2005^{(14)}$. A escala compreende 22 perguntas classificadas em três domínios, sendo eles a autoeficácia para controle da dor (aborda como o paciente controla sintomas físicos como a fadiga e a dor), a autoeficácia para funcionalidade (aborda a realização de algumas atividades diárias) e a autoeficácia para lidar com outros sintomas (aborda 
a maneira como a dor afeta o indivíduo). Em cada domínio é possível obter uma pontuação máxima de 100 e mínima de 10. A pontuação total máxima a ser obtida na escala é de 300 e a mínima de 30, sendo que quanto maior a pontuação, maior o senso de autoeficácia.

Para avaliar a qualidade de vida, utilizou-se a versão resumida do WHOQOL-100, instrumento construído pela Organização Mundial da Saúde e validado para a língua portuguesa ${ }^{(15)}$, o WHOQOL-bref. $O$ WHOQOL-bref é um instrumento composto por 26 questões que abrangem um domínio geral e quatro domínios específicos, os domínios físico, psicológico, relações sociais e meio ambiente. O método utilizado para a obtenção das respostas é o de categorias, cada questão possui uma escala com respostas, que variam de um a cinco. Os escores de qualidade de vida para cada domínio e para a avaliação geral variam de zero a 100 , sendo que quanto maior o valor, melhor é o nível de qualidade de vida ${ }^{(15)}$.

Para análise e caracterização dos dados foi utilizada a estatística descritiva com medidas de tendência central (média, mínimo e máximo) e medida de dispersão (desvio-padrão), além de cálculos de frequência simples e percentual.

A qualidade de vida investigada por WHOQOL - Bref foi analisada por meio da proposta de análise feita pelo Grupo WHOQOL no site http://www.ufrgs.br/psiquiatria/psiq/whoqol86.html.

O teste formal de Shapiro-Wilk foi realizado com 95\% de confiança, para verificar a suposição de normalidade dos dados, sendo que o resultado de P-valor foi menor que 0,05. Desta forma, utilizou-se a técnica não paramétrica de correlação de Spearman para a análise das correlações entre as variáveis. 0 coeficiente $\rho$ de Spearman mede a intensidade da relação entre variáveis ordinais; foi utilizado o nível de significância de $0,05^{(16)}$.

\section{RESULTADOS}

A amostra constou de 120 pessoas selecionadas por meio do prontuário médico, no entanto, contando as exclusões e perdas, participaram do estudo 95 pessoas com dor crônica.

As mulheres representam a maioria dos entrevistados. A média de idade encontrada foi de 55 anos $(d p=11,4)$, com variação entre 29 e 91 anos. A faixa etária de maior prevalência foi entre pessoas com menos de 60 anos. Grande parte da amostra se classifica como sendo de cor branca, com predomínio de pessoas com ensino fundamental incompleto. $\mathrm{O}$ sobrepeso e a obesidade foram encontrados com alta prevalência nesta amostra (Tabela 1).

Com relação à localização da dor, ressalta-se que a maioria dos participantes da pesquisa apresenta dor crônica em mais de um local do corpo $(71,60 \%)$, sendo que a maior prevalência foi de dor lombar $(32,50 \%)$, dor nos membros inferiores $(29,0 \%)$, membros superiores $(23,50 \%)$, região cervical $(8,00 \%)$, região torácica $(5,50 \%)$ e cabeça $(1,50 \%)$.

As principais causas de dor crônica encontradas foram artrose $(40,20 \%)$, osteofitose $(15,60 \%)$, hérnia de disco $(11,40 \%)$, escoliose/lordose $(9,90 \%)$, fibromialgia $(9,00 \%)$ e artrite $(4,00 \%)$. 
Tabela 1: Frequência dos indivíduos com dor crônica em uma Unidade de Saúde da Família, segundo variáveis sociodemográficas e IMC. São Carlos, SP, Brasil, 2010.

\begin{tabular}{|c|c|c|}
\hline \multicolumn{2}{|c|}{ Variáveis } & \multirow{2}{*}{$\begin{array}{c}\mathbf{N}(\%) \\
76(80 \%)\end{array}$} \\
\hline \multirow{2}{*}{ Gênero } & Feminino & \\
\hline & Masculino & $19(20 \%)$ \\
\hline \multirow{2}{*}{ Idade } & $<60$ anos & $64(67,37 \%)$ \\
\hline & $>60$ anos & $31(32,63 \%)$ \\
\hline \multirow{4}{*}{ Cor da pele } & Branca & $79(83,16 \%)$ \\
\hline & Negra & $05(5,26 \%)$ \\
\hline & Parda & $10(10,53 \%)$ \\
\hline & Amarela & $01(1,05 \%)$ \\
\hline \multirow{7}{*}{ Escolaridade } & Analfabeto & $01(1,05 \%)$ \\
\hline & Fundamental Incompleto & $46(48,42 \%)$ \\
\hline & Fundamental Completo & $16(16,84 \%)$ \\
\hline & Médio Incompleto & $02(2,11 \%)$ \\
\hline & Médio Completo & $22(23,16 \%)$ \\
\hline & Superior Incompleto & $01(1,05 \%)$ \\
\hline & Superior Completo & $07(7,37 \%)$ \\
\hline \multirow{3}{*}{ Renda Familiar (salário mínimo)* } & Até 2 & $30(31,58 \%)$ \\
\hline & 2 a 4 & $42(44,21 \%)$ \\
\hline & $>4$ & $23(24,21 \%)$ \\
\hline \multirow{4}{*}{ IMC** } & Baixo Peso $(<18,5)$ & $02(2,11 \%)$ \\
\hline & Peso Normal $(18,6$ a 24,9$)$ & $33(34,73 \%)$ \\
\hline & Sobrepeso (25 a 29.9) & $31(32,63 \%)$ \\
\hline & Obesidade (>30) & $29(30,53 \%)$ \\
\hline
\end{tabular}

* Salário Mínimo - Brasil, 2010 (SM): R\$ 560,00

** Índice de Massa Corporal - IMC. Para classificação foi utilizada a classificação da organização Mundial da Saúde ${ }^{(11)}$.

A média dos escores de intensidade da dor percebida foi de 6,90 pontos. 0 escore total médio de autoeficácia para dor crônica foi de 185,47 pontos, sendo que a maior pontuação entre os domínios da Escala de Autoeficácia foi encontrada no domínio funcionalidade. O domínio controle da dor foi o mais prejudicado. O escore médio total de qualidade de vida observado foi de 56,31 pontos. O domínio com menor pontuação, ou seja, que ocasiona maior impacto para a qualidade de vida desta amostra foi o domínio psicológico com média de 61,64 pontos (Tabela 2).

Tabela 2: Escores obtidos de uma amostra de indivíduos com dor crônica segundo intensidade de dor, domínios da Escala de autoeficácia para dor crônica e domínios da escala de qualidade de vida - WHOQOL-bref. São Carlos, SP, Brasil, 2010.

\begin{tabular}{cccccc}
\hline & Variáveis & Média & Mín. & Máx. & Desvio Padrão \\
\hline \multirow{4}{*}{ Autoeficácia } & Intensidade de dor & 6,90 & 2 & 10 & 2,23 \\
& Controle da dor & 50,28 & 10 & 100 & 22,29 \\
& Funcionalidade & 73,84 & 10 & 100 & 25,25 \\
& Lidar com outros sintomas & 61,33 & 11,25 & 100 & 21,82 \\
& Total & 185,47 & 48,25 & 294,40 & 58,21 \\
\multirow{2}{*}{ Qualidade de vida } & Domínio Físico & 63,63 & 17,85 & 100 & 17,88 \\
& Domínio Psicológico & 61,64 & 16,66 & 100 & 19,93 \\
& Domínio Relações Sociais & 67,01 & 16,66 & 100 & 18,31 \\
& Domínio Meio Ambiente & 66,08 & 31,25 & 100 & 15,28 \\
\end{tabular}

As correlações entre os domínios da autoeficácia e a intensidade de dor apresentaram correlações negativas de fracas a moderadas $(p<0,05)$. As correlações entre os domínios da qualidade de vida e a intensidade de dor apresentaram correlações negativas de fracas a moderadas $(p<0,05)$. As correlações entre 
os domínios da autoeficácia e os domínios da qualidade de vida apresentaram correlações positivas, fracas a moderadas, sendo as maiores correlações entre o domínio físico e psicológico da qualidade de vida e os domínios da autoeficácia, sugerindo que melhores níveis de autoeficácia resultam em melhores níveis de qualidade de vida, principalmente nos domínios físico e psicológico $(p<0,05)$ (Tabela 3).

Tabela 3: Correlações de Spearman entre intensidade de dor, domínios e valor geral da autoeficácia e domínios e valor geral da qualidade de vida. São Carlos, SP, Brasil, 2010.

\begin{tabular}{ccccccc}
\hline Variáveis & Intensidade & Físico & Psicológico & Relações Sociais & Meio Ambiente & Geral \\
\hline Intensidade & 1 & $-0,35$ & $-0,27$ & $-0,22$ & $-0,32$ & $-0,26$ \\
Controle da dor & $-0,13$ & 0,53 & 0,38 & 0,26 & 0,27 & 0,39 \\
Funcionalidade & $-0,33$ & 0,56 & 0,31 & 0,12 & 0,27 & 0,33 \\
Lidar com outros sintomas & $-0,32$ & 0,64 & 0,55 & 0,26 & 0,49 & 0,53 \\
Geral & $-0,32$ & 0,69 & 0,49 & 0,28 & 0,39 & 0,48 \\
\hline
\end{tabular}

Desta forma, os resultados apontam que indivíduos com dor crônica e com elevados níveis de autoeficácia podem apresentar menor intensidade de dor e melhor qualidade de vida, principalmente nos domínios físico e psicológico, e ainda, que baixos índices de autoeficácia ocasionam diminuição da qualidade de vida.

\section{DISCUSSÃO}

Na presente pesquisa constatou-se que indivíduos com dor crônica que apresentam altos índices de autoeficácia conseguem controlar melhor seus sintomas dolorosos. E ainda, que as correlações entre os domínios da autoeficácia e da qualidade de vida foram todas positivas. Tais resultados foram encontrados em outros estudos ${ }^{(2,8,17-18)}$.

$\mathrm{Na}$ análise da relação entre as variáveis autoeficácia e qualidade de vida com a intensidade de dor, o domínio funcionalidade da Escala de Autoeficácia e o domínio físico do WHOQOL-bref foram os domínios que apresentaram maiores níveis de correlação negativa com a intensidade da dor, o que demonstra o quanto a intensidade de dor afeta a dimensão física da pessoa acometida, impedindo muitas vezes a realização de atividades do dia a dia. Cabe ressaltar que na Escala de Autoeficácia o domínio funcionalidade se refere às crenças que a pessoa tem de que é capaz de realizar as atividades diárias e funcionais.

As crenças de autoeficácia associam-se com incapacidade ${ }^{(2)}$. Em outro estudo de corte transversal constataram que a autoeficácia associou-se com qualidade de vida, sendo que a qualidade de vida está associada aos altos níveis de autoeficácia dos indivíduos acometidos por dor crônica ${ }^{(17)}$. E ainda, que as pessoas com dor crônica que apresentam um nível alto de autoeficácia e qualidade de vida satisfatória são mais influenciáveis para efetivar com sucesso o tratamento para dor, seja ele medicamentoso ou de mudança de estilo de vida ${ }^{(17)}$.

Resultados de um estudo espanhol realizado em indivíduos com fibromialgia ${ }^{(9)}$ apontaram a grande potencialidade terapêutica da autoeficácia. Neste estudo, os autores ainda sinalizaram que inserir a avaliação 
da autoeficácia não só produzirá efeitos benéficos em diferentes cenários do cuidado, mas também favorecerá um processamento menos catastrofista da informação dolorosa, que por sua vez, terá efeitos positivos para o impacto global da doença. A relação negativa entre autoeficácia e catastrofismo também foi evidenciada, sendo que as pessoas que se percebem autoeficazes no manejo da sua dor processam de forma menos catastrófica a situação dolorosa ${ }^{(9)}$.

A avaliação das crenças de autoeficácia pode ser utilizada como um meio para guiar as condutas dos profissionais da saúde no manejo da dor e, se necessário, desenvolver ou fortalecer tais crenças. Além disso, pode ser usada como uma ferramenta para avaliar os resultados após a conduta tomada para o manejo da dor crônica ${ }^{(2)}$. A avaliação da qualidade de vida para o manejo da dor crônica também se torna de extrema importância, pois, ao se avaliarem os domínios da qualidade de vida (físico, emocional, social e ambiental), é possível direcionar o foco do manejo para o aspecto de maior comprometimento ${ }^{(17-18)}$.

Um estudo ${ }^{(19)}$ apontou que a incapacidade é influenciada pelas crenças de autoeficácia, indicando que indivíduos com baixos níveis de autoeficácia não se envolvem efetivamente no tratamento, tendem a ter atitude mais passiva e desistem facilmente de seus objetivos na presença de obstáculos. Por outro lado, indivíduos com autoeficácia elevada aderem melhor ao tratamento, tendem a ser mais persistentes e, em geral, mantêm a maior parte de suas atividades, apesar da dor.

Autores de um estudo longitudinal concluíram que a autoeficácia é uma variável mais importante do que o medo do movimento para mediar a relação entre dor e incapacidade em pessoas com dor lombar crônica ${ }^{(20)}$.

A avaliação das crenças de autoeficácia pode ser utilizada como um meio para guiar as condutas e, se necessário, determinar e incentivar a autoconfiança dos pacientes em realizar atividades normais. Além disso, pode ser usada como uma ferramenta para avaliar os resultados após o manejo da dor crônica ${ }^{(18-22)}$. A avaliação da qualidade de vida para o manejo da dor crônica também se torna de extrema importância, pois, ao se avaliarem os domínios da qualidade de vida (físico, emocional, social e ambiental), é possível direcionar o foco do manejo para o aspecto de maior comprometimento ${ }^{(17)}$.

Uma avaliação adequada do indivíduo com dor crônica inclui conhecer suas potencialidades e suas fraquezas. Identificar pessoas com baixos níveis de autoeficácia e intervir no fortalecimento dessas crenças pode ser uma estratégia bastante eficaz para melhorar os resultados do manejo da dor crônica. O uso de intervenções para proporcionar a autogestão da dor pode ser uma estratégia do enfermeiro para a melhora da autoeficácia à medida que empodera o indivíduo e lhe dá forças para gerir seus sintomas.

Cabe ressaltar que as limitações do presente estudo são o fato de o número amostral ter sido por conveniência, sendo que os participantes inseridos na amostra foram apenas aqueles que tinham, no prontuário cadastrado na Unidade de Saúde da Família estudada, o diagnóstico médico de alguma doença que causasse dor crônica. 


\section{CONCLUSÃO}

Os resultados permitem concluir que indivíduos com dor crônica e elevados níveis de autoeficácia podem apresentar menor intensidade de dor e melhor qualidade de vida, e ainda, que baixos índices de autoeficácia ocasionam diminuição da qualidade de vida. Este estudo propõe que a avaliação de enfermagem do indivíduo com dor crônica deva incluir a avaliação da autoeficácia e da qualidade de vida para direcionar o planejamento da assistência, visto que conhecer as potencialidades e as fraquezas do indivíduo possibilita pensar no uso de estratégias para desenvolver/fortalecer a autoeficácia.

\section{REFERÊNCIAS}

1. Keefe FJ, Porter L, Somers T, Shelby R, Wren AV. Psychosocial interventions for managing pain in older adults: outcomes and clinical implications. Br J Anaesth [Internet]. 2013 [acesso em:31 Mar. 2016];111(1):89-94. Disponível em: http://dx.doi.org/10.1093/bja/aet129.

2. Sardá Júnior JJ, Nicholas MK, Pimenta CAM, Asghari A. Biopsychosocial predictors of pain, incapacity and depression in Brazilian chronic pain patients. Rev Dor [Internet]. 2012 [acesso em: 31 Mar. 2016];13(2):111-8. Dísponível em: http://dx.doi.org/10.1590/S1806-00132012000200003.

3. Miles CL, Pincus T, Carnes D, Taylor SJ, Underwood M. Measuring pain self-efficacy. Clin J Pain [Internet]. 2011 [acesso em: 31 Mar. 2016];27(5):461-70. Disponível em: http://dx.doi.org/10.1097/AJP.0b013e318208c8a2.

4. Bandura A. Self-efficacy: The exercise of control. New York: Freeman; 1997.

5. Mann EG, Lefort S, Vandenkerkhof EG. Self-management interventions for chronic pain. Pain Manag [Internet]. 2013 [acesso em: 31 Mar. 2016];3(3):211-22. Dísponível em: http://dx.doi.org/10.2217/pmt.13.9.

6. McBeth J, Prescott G, Scotland G, Lovell K, Keeley P, Hannaford P, et al. Cognitive behavior therapy, exercise, or both for treating chronic widespread pain. Arch Intern Med [Internet]. 2012 [acesso em: 31 Mar. 2016];172(1):48-57. Disponível em: http://dx.doi.org/10.1001/archinternmed.2011.555.

7. World Health Organization. WHOQOL. Measuring Quality of Life [Internet]. Genebra: WHO; 1997 [acesso em: 31 Mar. 2016]. Disponível em: http://www.who.int/mental_health/media/68.pdf.

8. Sardá Júnior J, Nicholas MK, Asghari A, Pimenta CA. The contribution of self-efficacy and depression to disability and work status in chronic pain patients: a comparison between Australian and Brazilian samples. Eur J Pain [Internet]. 2009 [acesso em: 31 Mar. 2016];13(2):189-95. Disponível em: http://dx.doi.org/10.1016/j.ejpain.2008.03.008.

9. Zafra-Polo MT, Pastor-Mira MA, López-Roig S. Autoeficacia, Catastrofismo, Miedo al Movimiento y resultados de salud en la Fibromialgia. An Psicol [Internet]. 2014 [acesso em: 31 Mar. 2016];30(1):104-13. Disponível em:

http://dx.doi.org/10.6018/analesps.30.1.151541.

10. Merskey H, Bogduk N. Classification of chronic pain: descriptions of chronic pain syndromes and definitions of pain terms [Internet]. Seattle: IASP Pain Terminology; 1994 [acesso em: 31 Mar. 2016]. Disponível em: http://www.iasppain.org/files/Content/ContentFolders/Publications2/FreeBooks/Classification-of-Chronic-Pain.pdf.

11. World Health Organization. Global Database on Body Mass Index. BMI Classification [Internet]. 2008 [acesso em: 31 Mar. 2016]. Disponível em: http://www.who.int/bmi/index.jsp?introPage=intro_3.html.

12. Bonica JJ. The management of pain. Lea e Febiger. 1990.

13. Anderson KO, Dowds BN, Pelletz RE, Edwards WT, Peeters-Asdourian C. Development and initial validation of a scale to measure self-efficacy beliefs in patients with chronic pain. Pain [Internet]. 1995 [acesso em: 31 Mar.

2016];63(1):77-84. Disponível em: http://dx.doi.org/10.1016/0304-3959(95)00021-J.

14. Salvetti MG, Pimenta CAM. Validação da Chronic Pain Self-Efficacy Scale para a língua portuguesa. Rev Psiquiatr Clínica [Internet]. 2005 [acesso em: 31 Mar. 2016];32(4):202-10. Disponível em: http://dx.doi.org/10.1590/S010160832005000400002.

15. Fleck MP, Louzada S, Xavier M, Chachamovich E, Vieira G, Santos L, et al. Aplicação da versão em português do instrumento abreviado de avaliação da qualidade de vida "WHOQOL-bref". Rev Saude Publica [Internet]. 2000 [acesso em: 31 Mar. 2016];34(2):178-83. Disponível em: http://dx.doi.org/10.1590/S0034-89102000000200012.

16. Siegel S, Castellan JN. Estatística não paramétrica para ciência do comportamento. 2a ed. Porto Alegre: Artmed; 
2006.

17. Yazdi-Ravandi S, Taslimi Z, Jamshidian N, Saberi H, Shams J, Haghparast A. Prediction of Quality of life by SelfEfficacy, Pain Intensity and Pain Duration in Patient with Pain Disorders. Basic Clin Neurosci [Internet]. 2013 [acesso em: 31 Mar. 2016];4(2):117-24. Disponível em: http://www.ncbi.nlm.nih.gov/pmc/articles/PMC4202536/.

18. Gormsen L, Rosenberg R, Bach FW, Jensen TS. Depression, anxiety, health-related quality of life and pain in patients with chronic fibromyalgia and neuropathic pain. Eur J Pain [Internet]. 2010 [acesso em: 31 Mar. 2016];14(2):127.e1-8. Disponível em: http://dx.doi.org/10.1016/j.ejpain.2009.03.010.

19. Salvetti MG, Pimenta CAM, Braga PE, Corrêa CF. Disability related to chronic low back pain: prevalence abd associated factors. Rev Esc Enferm USP [Internet]. 2012 [acesso em: 31 Mar. 2016];46(esp):16-23. Disponível em: http://dx.doi.org/10.1590/S0080-62342012000700003.

20. Costa LC, Maher CG, McAuley JH, Hancock MJ, Smeets RJ. Self-efficacy is more important than fear of movement in mediating the relationship between pain and disability in chronic low back pain. Eur J Pain [Internet]. 2011 [acesso em: 31 Mar. 2016];15(2):213-9. Disponível em: http://dx.doi.org/10.1016/j.ejpain.2010.06.014. 